\title{
Interface Synthesis using Memory Mapping for an FPGA Platform
}

Manev Luthra ${ }^{\ddagger}$ Sumit Gupta ${ }^{\ddagger}$ Nikil Dutt ${ }^{\ddagger}$ Rajesh Gupta ${ }^{\S}$ Alex Nicolau

\author{
CECS \\ Technical Report \#03-20 \\ June 2003
}

\section{Center for Embedded Computer Systems}

${ }^{\sharp}$ School of Information and Computer Science ${ }^{\S}$ Dept. of Computer Science and Engineering

University of California at Irvine

\{mluthra, sumitg, dutt, nicolau\}@ cecs.uci.edu
University of California at San Diego

gupta@cs.ucsd.edu

http://www.cecs.uci.edu/ spark 


\begin{abstract}
Several system-on-chip (SoC) platforms have recently emerged that use reconfigurable logic (FPGAs) as a programmable co-processor to reduce the computational load on the main processor core. We present an interface synthesis approach that enables us to do hardware-software codesign for such FPGA-based platforms. The approach is based on a novel memory mapping algorithm that maps data used by both the hardware and the software to shared memories on the reconfigurable fabric. The memory mapping algorithm uses scheduling information from a high-level synthesis tool to map variables, arrays and complex data structures to the shared memories in a way that minimizes the number of registers and multiplexers used in the hardware interface. We also present three software schemes that enable the application software to communicate with this hardware interface. We demonstrate the utility of our approach and study the trade-offs involved using a case study of the co-design of a computationally expensive portion of the MPEG-1 multimedia application on to the Altera Nios platform.
\end{abstract}




\section{Contents}

1 Introduction $\quad 4$

2 Related Work $\quad 5$

3 Role of Interface Synthesis and Memory Mapping in a Co-Design Methodology 5

3.1 Memory Mapping . . . . . . . . . . . . . . . . . . . . . . . . . . 6

3.2 Problem Formulation $\ldots \ldots \ldots \ldots \ldots \ldots \ldots \ldots$

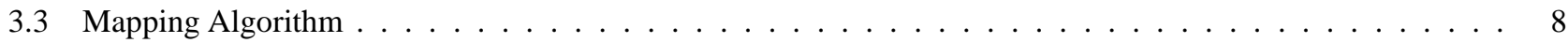

3.4 Construction of Conflict Graphs $\ldots \ldots \ldots \ldots \ldots \ldots \ldots \ldots \ldots \ldots$

4 The Hardware Interface Architecture 12

5 The SW Interface $r$

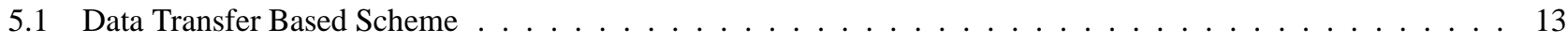

5.2 Shared Memory Based Schemes $\ldots \ldots \ldots \ldots \ldots \ldots \ldots \ldots \ldots \ldots$

6 Experimental Setup and Results $\quad 14$

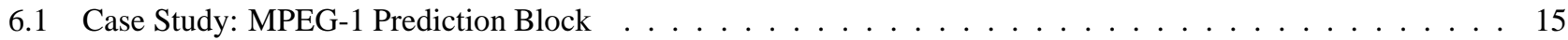

7 Conclusions and Future Work $\quad 18$ 


\section{List of Figures}

1 Role of interface synthesis in a co-design methodology . . . . . . . . . . . . . . . . 6

2 The advantages of memory mapping . . . . . . . . . . . . . . . . . . . 7

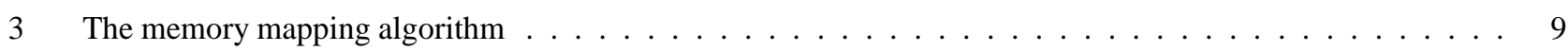

4 Determining the list of available memories . . . . . . . . . . . . . . . . . . . . 10

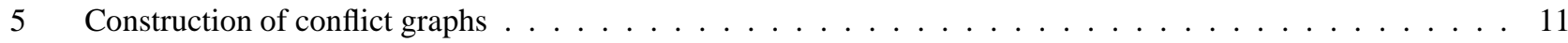

6 The hardware interface architecture . . . . . . . . . . . . . . . . . . . 12

7 Modified address offsets in the software interface after memory mapping . . . . . . . . . . . . 13

8 The Nios embedded system . . . . . . . . . . . . . . . . . . . . . . . . . . . .

9 Execution times for different hardware software partitions of the application . . . . . . . . . . . . 18

\section{List of Tables}

1 Logic synthesis results for the target FPGA . . . . . . . . . . . . . . . . . . 16

2 Execution times of various kernels of the application . . . . . . . . . . . . . . . . . 17 


\section{Introduction}

Platform based designs provide promising solutions for handling the growing complexity of chip designs [1]. FPGAs often play an important role in platforms by providing flexible, reconfigurable circuit fabrics to build and optimize target applications.

Our focus is on platform designs for multimedia and image processing applications. Our target architecture models many emerging platforms that contain a general purpose processor assisted by dedicated hardware for computationally intensive tasks. The hardware assists are implemented on-board FPGA blocks and thus, can be programmed for different applications. Binding the application functionality to software and hardware requires automated methods to specify, generate and optimize the interface between them. The interface should be fast, transparent and require minimal hardware and software resources. This report presents a methodology to generate these interfaces.

Multimedia and image processing applications typically operate on a large data set. Consequently, when these applications are partitioned and mapped onto an FPGA-based platform, this data has to be communicated and shared between the hardware and software components. Configurable logic blocks in FPGAs are typically inefficient for use as memories: if we store each data element in a register and provide an independent access mechanism for each one, then the resulting memory implementation occupies a large portion of the FPGA fabric. Instead, an efficient way to implement these large memories is to cluster the data elements into RAMs or register banks. In this report, we present our interface synthesis approach that efficiently utilizes embedded RAMs in FPGAs to implement the memory. Our approach is based on a novel memory mapping algorithm that generates and optimizes a hardware interface used for integrating the computationally expensive application kernels (hardware assists) with the rest of the platform.

Our memory mapping algorithm makes use of scheduling information on per cycle data access patterns (available from the high-level synthesis tool) in order to map registers to memories. The unique feature of this algorithm is its ability to efficiently handle designs in which data access patterns are unknown during scheduling - for example, an array being indexed by variable indices which become known only at run-time. This feature proves to be extremely useful when dealing with designs involving control flow.

To validate our co-design methodology, we present a case study of the co-design of a computationally expensive portion of the MPEG-1 multimedia application. We find that without using our memory mapping algorithm, the portion mapped to the FPGA is too big to fit inside it. We also compare results for various hardware-software interfacing schemes used with this design.

The rest of this report is organized as follows: in the next section, we discuss related work. In Section 3, 
we describe our methodology and the relation between memory mapping and hardware interface synthesis. We also formulate the memory mapping problem and present an algorithm for solving it. In Section 4 we describe the architecture of our hardware interface. In Section 5, we describe the changes required in the software to use the hardware interface and explain three hardware-software interfacing schemes. In Section 6, we describe our MPEG-1 case study and then, conclude the report with a discussion.

\section{Related Work}

Hardware-software partitioning [2,3] and high level synthesis [4, 5] have received significant attention over the past decade. Interface synthesis techniques have focused on various issues like optimizing the use of external IO pins of micro-controllers and minimizing glue logic [6]. However, the use of memory mapping for interface synthesis has not been considered. Furthermore, hardware-software co-design methodologies that synthesize the hardware component as an ASIC, pay little attention towards optimizing the memory mapping since the amount of logic that can be mapped to an ASIC is less severely constrained than that for FPGAs $[2,7,8]$.

Most previous work on memory mapping and allocation of multiport memories has been done in the context of data path synthesis and has focused on purely data flow designs (no control constructs) [9, 10, 11]. These algorithms do not deal with unknown data access patterns because no control flow is involved. Memory mapping and register binding algorithms in the data path synthesis domain are based on variable lifetime analysis and register allocation heuristics $[10,12,13]$.

Early work on memory mapping in the context of FPGAs has not utilized scheduling information [12, 14]. Karchmer and Rose present an algorithm for packing data structures with different aspect ratios into fixed width memories available on FPGAs [15]. However, this is of limited use when applications use simple and regular data structures.

\section{Role of Interface Synthesis and Memory Mapping in a Co-Design Methodology}

Interface synthesis is an important aspect of our hardware-software co-design methodology, as shown in Figure 1. In our approach, we rely on a $\mathrm{C} / \mathrm{C}++$ based description $[16,17,18]$ for the system model. After hardwaresoftware partitioning, the hardware part is scheduled using a high-level synthesis tool and the scheduling information is passed to the interface synthesizer.

This interface synthesizer - described in detail in the rest of the report - generates the hardware interface and re-instruments the software component of the application to make appropriate calls to the hardware component via this interface. It also passes the addresses of all registers that have been mapped to memories in the hardware interface to the high-level synthesis tool. 


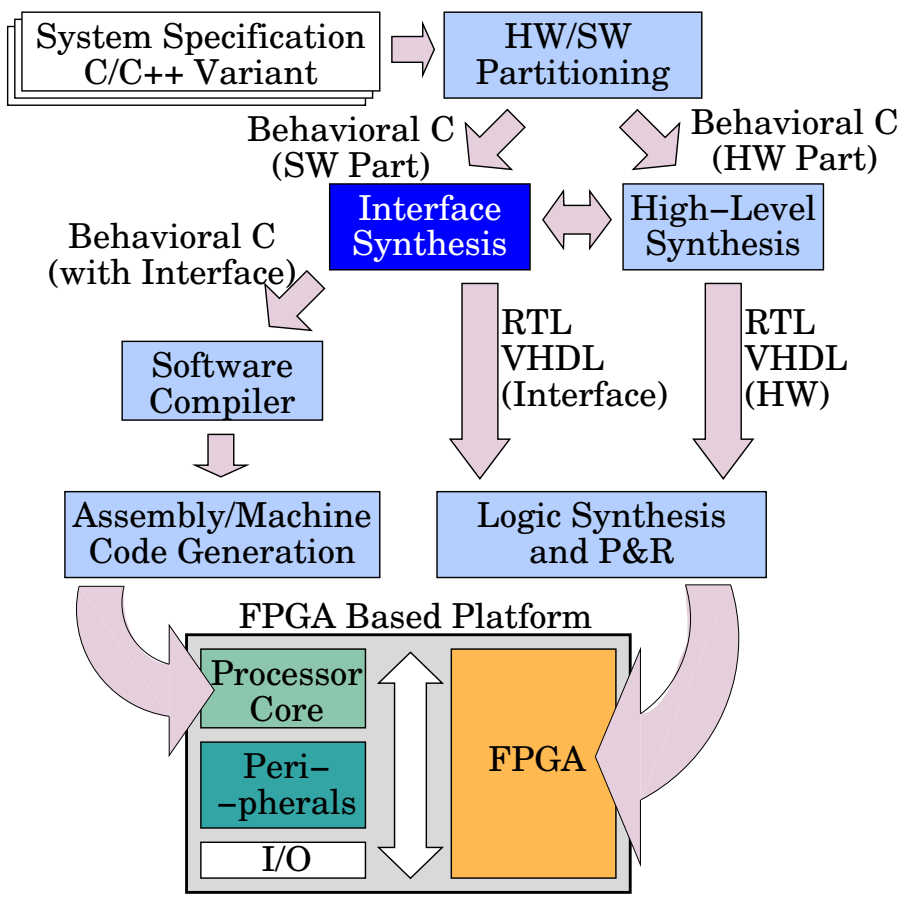

Figure 1. Role of interface synthesis in a co-design methodology

The RTL code generated by the high-level synthesis tool and the interface synthesizer are then downloaded to the FPGA on the platform. Similarly, the software component is compiled and downloaded into the instruction memory of the processor.

\subsection{Memory Mapping}

Multimedia and image processing applications process large amounts of data. After partitioning, the hardware component has to operate on the same data that the software operates on. Thus, the hardware component needs to store this data on the FPGA (see Section 4 for how this is achieved). Also, the stored data has to be multiplexed and steered to various functional units that comprise the hardware component. The presence of control flow in the application code also adds significantly to the multiplexing costs.

The way the data is mapped to the memory has a tremendous impact on the complexity of the multiplexers and control generated. Ideally, we would store all data in a single large memory. However, such a memory would require as many ports as the maximum number of simultaneous memory accesses in any cycle [11]. This is impractical for programmable FPGA platforms, since they provide memories with only a limited number of ports $[19,20]$. Consequently, memories with a larger number of ports have to be implemented using individual registers. This requires a large number of registers and complex, large multiplexers as shown in Figure 2(a).

In our memory mapping approach, we utilize scheduling information - available from the high-level synthesis 


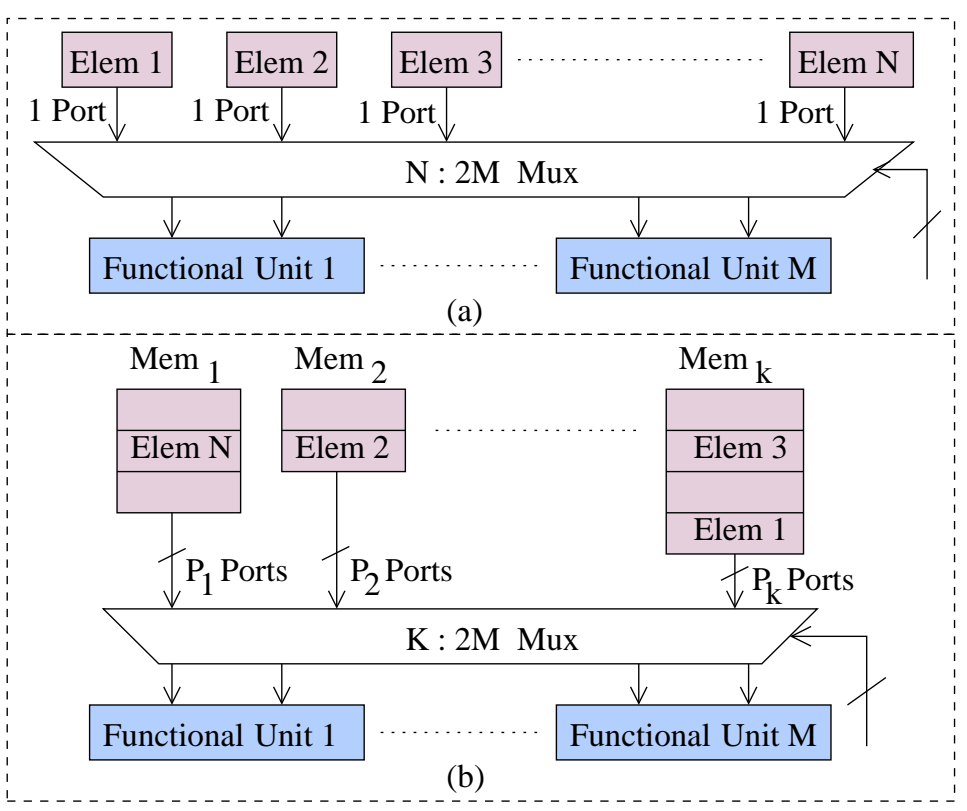

Figure 2. (a) Unmapped Design: Registers for each data element (b) Mapped Design: Data elements mapped to memories, $K \ll N$

tool - about data accesses and the cycles that they occur in. We can then map the data elements to memory banks, given constraints on the maximum number of ports each memory in the target FPGA can have. This approach eliminates the use of registers for storage, thus, saving a large amount of area, which in turn can be used for the application logic. This way, we can also use much smaller and faster multiplexers in the data-path as illustrated in Figure 2(b). In this figure, $\operatorname{size}\left(\mathrm{Mem}_{1}\right)+\operatorname{size}\left(\mathrm{Mem}_{2}\right)+\ldots .+\operatorname{size}\left(\mathrm{Mem}_{k}\right)=N$.

Arrays and data structures are mapped to memories after being broken down into their basic constituents (variables). These can then be mapped in a way identical to regular variables. Consequently, these basic constituents might get mapped to non-contiguous memory addresses/locations. In Section 5 we show how this drawback can easily be overcome by making a few changes to the application software.

\subsection{Problem Formulation}

We are given a set of $n$ variables, $V=\left\{v_{i} ; i=1,2, \ldots, n\right\}$ that are accessed (read and written) by all the kernels of the application. In our current model, only one kernel executes at any given time. This implies that contention for variable accesses between two kernels can never occur. Note that, each element in an array or data structure is considered as a distinct variable $v_{i}$ in $V$; so for example, an array of size $n$ will have $n$ entries in $V$. We are also given a set of memory resource types, $M_{\text {type }}=\left\{m_{j} ; j \in Z^{+}\right\}$where the subscript $j$ indicates the maximum number of ports available. The number of read ports of memory type $m_{j}$ are given by $\operatorname{Ports}_{\text {read }}\left(m_{j}\right)$ and write ports by $\operatorname{Ports}_{\text {write }}\left(m_{j}\right)$. 
Definition 3.1 The memory mapping problem is to find a memory allocation $\phi:\left(M_{\text {types }} \times Z^{+}\right) \rightarrow V$ that is a mapping of memory instances to variables assigned to them in the design. This mapping also gives the list $M$ of all the memory instances allocated to the design. $\phi\left(m_{j}, n\right)$ represents the list of variables mapped to the $n$-th instance of memory type $m_{j}$. The optimization problem is to minimize the total number of memory instances, given by size $(M)$, with the constraint that for each memory instance $\left(m_{j}, n\right)$ used in the design, the number of simultaneous accesses during any cycle should not exceed the number of memory ports available on $m_{j}$.

\subsection{Mapping Algorithm}

The problem defined above is an extension of the memory mapping and value grouping problem for datapath synthesis, which is known to be NP-complete [21]. We adopt a heuristic approach to solving it; our memory mapping algorithm is listed in Figure 3. For each variable to be mapped to a memory instance, the algorithm calls the function GetList OfCandMems to get a list of candidate memory instances $(\mathcal{L})$ onto which the current variable $v_{c}$ can potentially be mapped (line 3 in Figure 3).

If this list is empty, a new memory instance with just enough ports for $v_{i}$ is created, and $v_{i}$ is mapped to it (lines 4 to 6). If the list is non-empty, we pick the memory instance with the lowest cost. If the number of ports available on this memory instance are sufficient to map $v_{i}$ to it, then $v_{i}$ is added to the list of variables $\phi\left(m_{j}, k\right)$ mapped to this instance; otherwise, a new memory instance $\left(m_{p}, q\right)$ with enough ports is created. The old memory instance $\left(m_{j}, k\right)$ is discarded after all variables mapped to it have been re-mapped to $\left(m_{p}, q\right)$. Finally, $v_{i}$ is mapped to $\left(m_{p}, q\right)$ (lines 9 to 13 in the algorithm).

The algorithm for the function GetListOfCandMems is listed in Figure 4. This algorithm considers each memory instance $\left(m_{j}, k\right)$ in $M$ already allocated to the design, and adds this instance to the list $\mathcal{L}$ of candidate memory instances if the variable $v_{c}$ can be mapped to $\left(m_{j}, k\right)$. A variable $v_{c}$ can be mapped to $\left(m_{j}, k\right)$ when, $v_{c}$ does not conflict in terms of reads or writes with any other variable mapped to $\left(m_{j}, k\right)$, or $\left(m_{j}, k\right)$ has enough ports for accessing variable $v_{c}$ besides all the variables already mapped to it (line 3 in Figure 4).

If $\left(m_{j}, k\right)$ does not have enough ports to map variable $v_{c}$, then we try to find a memory of type $m_{p}$, such that, an instance of $m_{p}$ will satisfy the port constraints when variables $v_{c}$ and $\phi\left(m_{j}, k\right)$ (variables already mapped to $\left(m_{j}, k\right)$ ) are mapped to it. If such a memory type exists, it marks memory instance $\left(m_{j}, k\right)$ for an upgrade to an instance of memory type $m_{p}(p>j)$ and adds it to $\mathcal{L}$ (lines 7 to 9 ).

The algorithm in Figure 4 also calculates a cost for mapping $v_{c}$ to each memory instance in $\mathcal{L}$. This cost equals the total number of read and write ports of the memory instance.

Assume that $A$ is the total number of hardware kernels accessing the memory, $s$ is the length of the longest schedule among these kernels, while $z$ is the maximum number of memory accesses occuring in a single cycle 


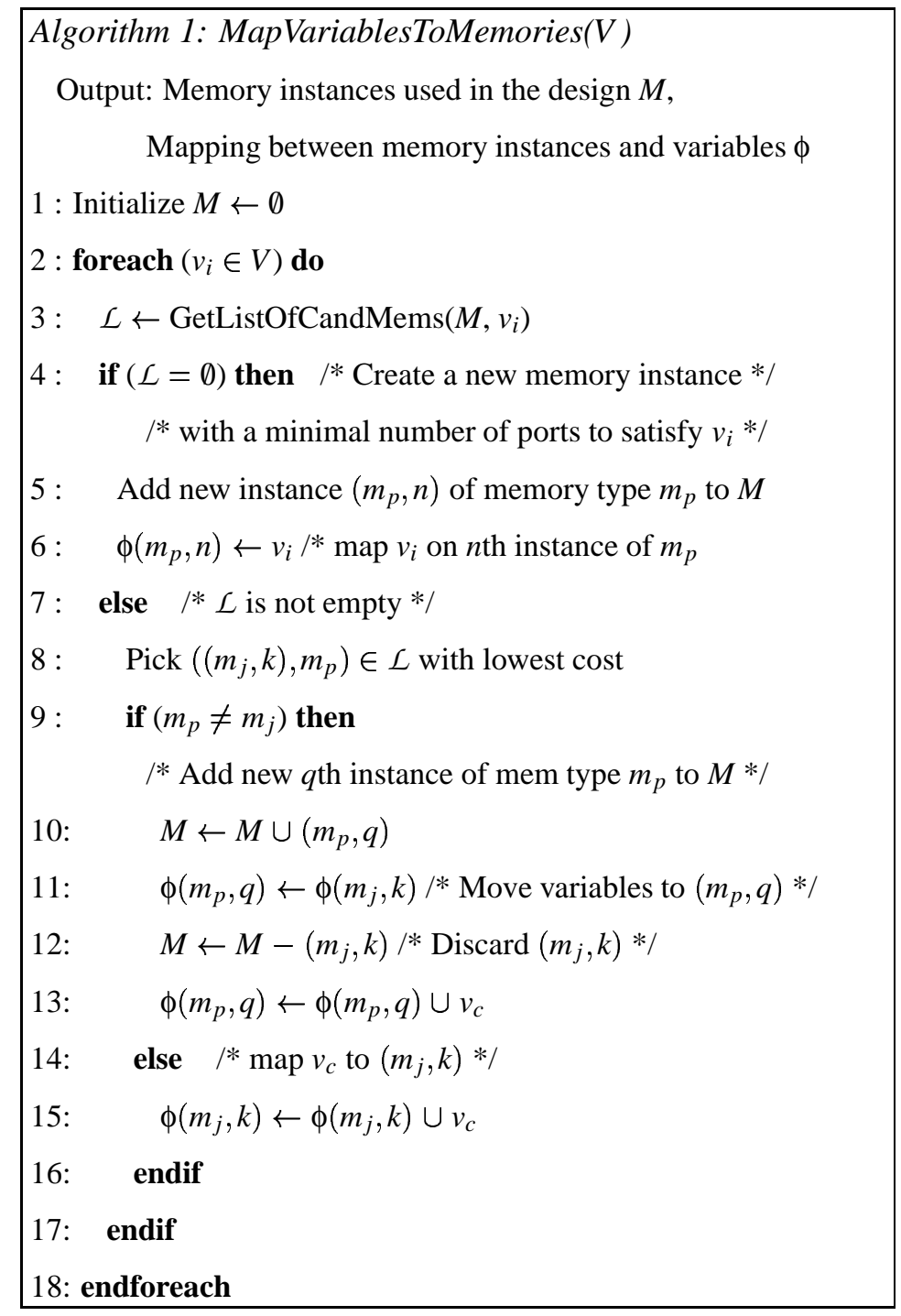

Figure 3. The memory mapping algorithm

by any one variable. Then, lines 2 and 3 in Figure 4 individually contribute $n$ and $A s z$ to the time complexity respectively. So the GetListofCandMems algorithm has a worst case time complexity of $O(n A s z)$. The loop in line 2 of the MapVariablesToMemories algorithm in Figure 3 causes the GetListofCandMems algorithm to execute $n$ times. Thus, the worst case time complexity of the MapVariablesToMemories algorithm is $O\left(n^{2} A s z\right)$.

\subsection{Construction of Conflict Graphs}

In the GetListOfCandMems algorithm, we determine if variable $v_{c}$ can be mapped to memory instance $\left(m_{j}, k\right)$ by checking for potential conflicts with the variables $\phi\left(m_{j}, k\right)$ that have already been mapped to $\left(m_{j}, k\right)$. This is done for every cycle and thus, we maintain conflict graphs for each cycle in the schedule. Nodes in conflict graphs represent variables and an edge between two nodes denotes a conflict between variables, i.e., both the variables 


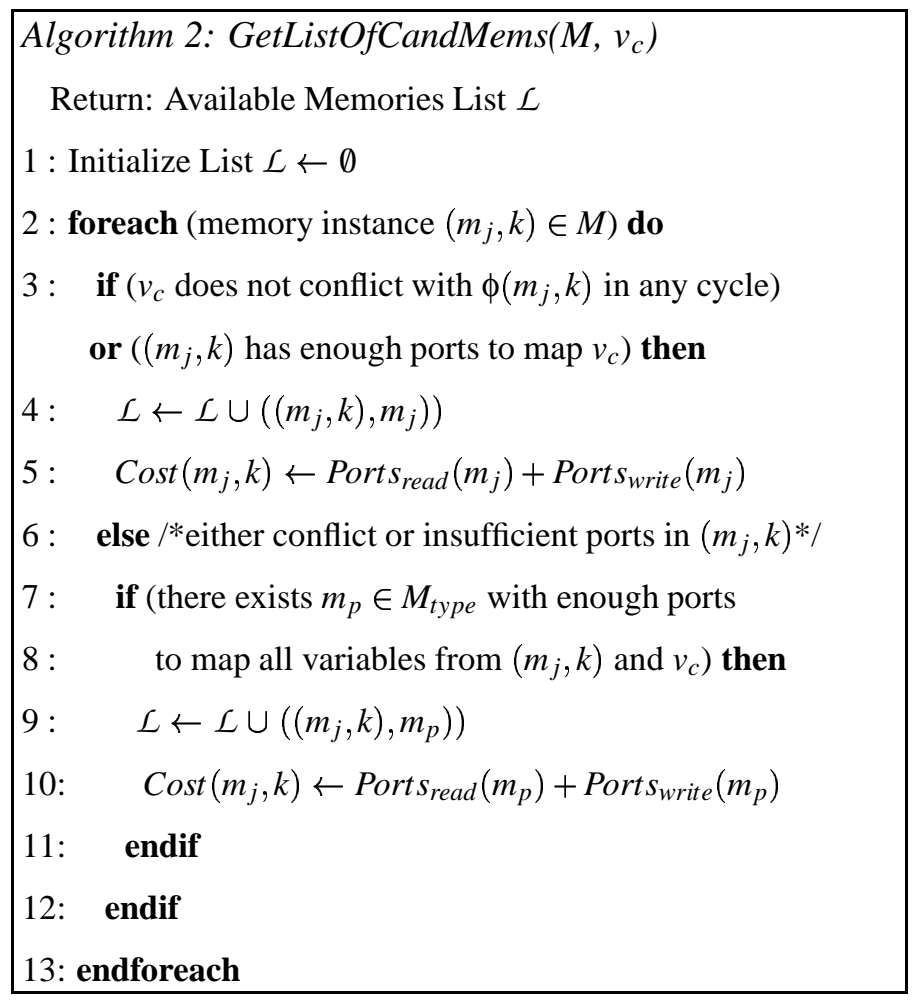

Figure 4. Determining the list of available memories

are accessed in that cycle.

To understand how we use these conflict graphs, consider a design with three variables $v_{1}, v_{2}$ and $v_{3}$. Assume that $v_{1}$ and $v_{2}$ are accessed during cycle 1 , while $v_{2}$ and $v_{3}$ are accessed during cycle 2 . The corresponding conflict graphs for the two cycles are given in Figures 5(a) and 5(b). If we have only one memory resource type, namely, a dual ported memory $m_{2}$, then, each of the three variables can be mapped to the same instance of the dual ported memory without violating the port constraints. This is because only two of the three variables conflict in any cycle. If we had represented this using a single conflict graph for all cycles, variable $v_{2}$ would not have been mapped to memory because two conflict edges would have been associated with it, even though the accesses occur in different cycles.

Let us explore further with another example. Consider an array arr consisting of three elements, $\operatorname{arr}[1], \operatorname{arr}[2]$ and $\operatorname{arr}[3]$. The corresponding variables in $V$ are $v_{1}, v_{2}$ and $v_{3}$. Also, assume that dual ported memories are the only memory types available. In any given cycle, if there are multiple accesses to arr using variable indices $i$ and $j$ (for example $\operatorname{arr}[i]$ and $\operatorname{arr}[j]$ ), then we cannot determine which elements of the array actually conflict until runtime. Hence, we create conflict edges between each pair of elements in arr in the conflict graph corresponding to that cycle. This results in the fully connected conflict graph shown in Figure 5(c). We can conclude from this 


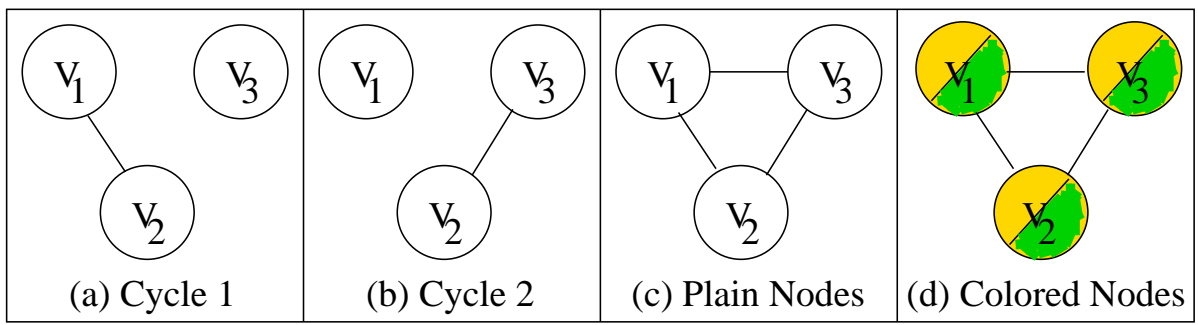

Figure 5. (a) Conflict graph for cycle 1 (b) Conflict graph for cycle 2 (c) Conflict graph with colorless nodes (d) Conflict graph with multi-colored nodes

conflict graph that none of the three variables can be mapped to the same memory instance since the memory has only two ports.

But, this is an incorrect conclusion because only two of the three variables will be accessed in any cycle. This implies that the three variables can be mapped to a dual ported memory. Thus, we find that per cycle conflict graphs are, by themselves, not powerful enough to capture all the information necessary to perform effective memory mapping.

To address such issues, we introduce the notion of accumulating colors in the nodes of the conflict graphs of each cycle. From among a group of variables $V_{G}$, if access of any one in a cycle rules out access of the rest in that cycle, then the nodes corresponding to each variable in the group $V_{G}$ are marked with the same color $c_{G}$. This color is unique from that of all other groups. A node can accumulate colors by being a member of more than one such group.

Applying this to our example, we get the conflict graph depicted in Figure 5(d). $v_{1}, v_{2}$ and $v_{3}$ form a group corresponding to access by $\operatorname{arr}[i]$. Each of these three variables are marked with one color since accessing any one of them rules out accessing the other two. Similarly, $v_{1}, v_{2}$ and $v_{3}$ form another group corresponding to access by $\operatorname{arr}[j]$ and are marked with a second color. Thus, each of the three nodes/variables end up accumulating two colors.

The number of ports needed to map a set of variables to a single memory instance, is equal to the maximum number of colors in any cycle of all the variables being mapped to that memory instance. In our example, the number of colors accumulated by $v_{1}, v_{2}$ and $v_{3}$ is two. Thus, we can safely map these three variables to a dual ported memory. We use this coloring technique while creating the per cycle conflict graphs used by our memory mapping algorithm. 


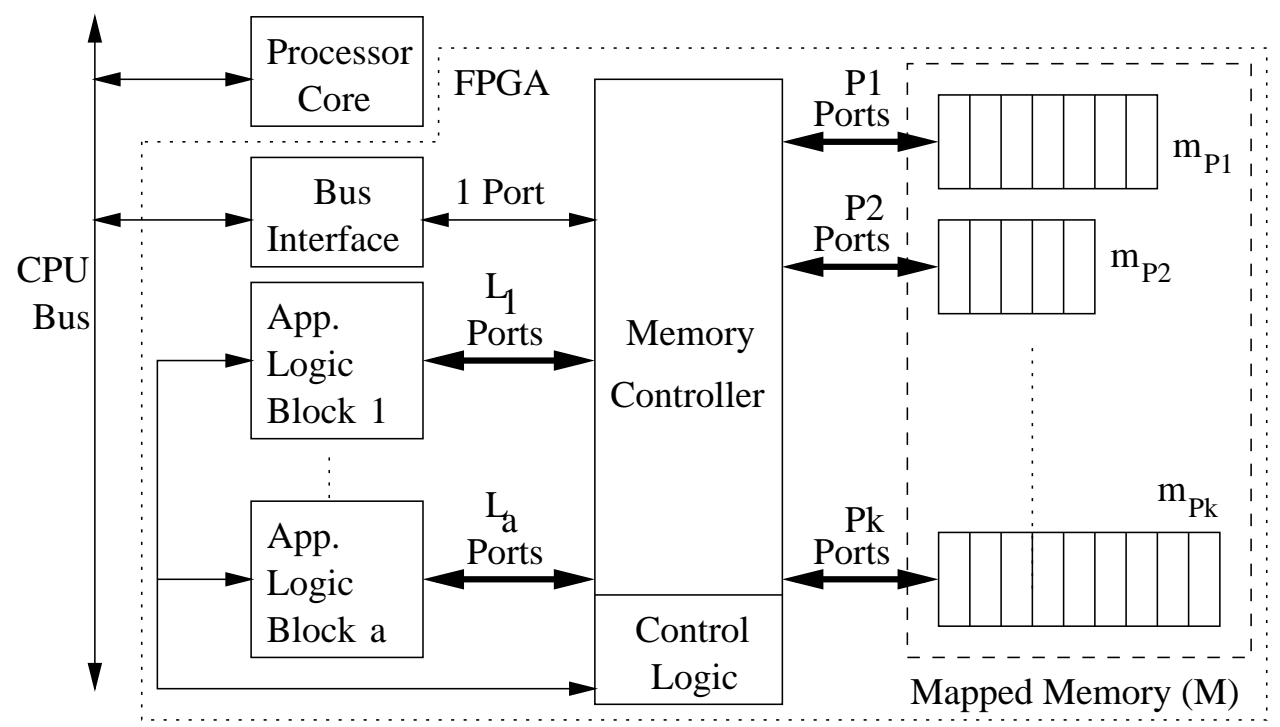

Figure 6. The hardware interface architecture

\section{The Hardware Interface Architecture}

An overview of the architecture obtained after hardware interface synthesis is shown in Figure 6. The bus interface generated is specific to the bus protocol used. The control logic contains memory mapped registers that can be used to reset or start/stop execution of any of the application logic blocks through software. It also contains registers reflecting the execution status and logic for interrupt generation, masking, et cetera.

The memory controller services all memory access requests for data residing in the mapped memory $M$. It is designed to give a higher priority to access requests by the application logic blocks. An access request by software is serviced only if a free port is currently available on the memory instance. Also, note that, although the memory controller can be designed to hide changes in the memory map (due to memory mapping) from software, we choose to make changes to the data structures in the application software instead. This is primarily because of implementation ease and the fact that, in any case, the application has to be re-instrumented to invoke the hardware. For very complex applications the best way might be to change both, i.e. hide only a few changes from software, while modifying the application appropriately to take care of the visible changes.

\section{The SW Interface}

In the hardware interface shown in Figure 6, the memory $M$ uses a contiguous address space. Hence, data declarations in the software code have to be reordered so that they conform to the order in which they were mapped to this address space. The example in Figure 7 illustrates this.

In order to make use of the hardware mapped to the FPGA, the software needs to interface and share data with 


\begin{tabular}{|ccc|}
\hline struct example \{ & & struct example \{ \\
int array1[256]; & & int element1; \\
int array2[256]; & New Memory & int array1[256]; \\
int element1; & Map & int element3; \\
int element2; & & int array2[256]; \\
int element3; & & int element2; \\
\} dm = 0xF800; & * dm = 0xF800;
\end{tabular}

Figure 7. Modified address offsets in the software interface after memory mapping

this hardware. The software can either transfer all the data to the hardware, or they can use a shared memory [8], as explained in the next two sections.

\subsection{Data Transfer Based Scheme}

In a data transfer scheme, all the shared data is copied from the processor (or main) memory to the mapped memory $M$ (in Figure 6). The hardware is then executed and the results are moved back from the mapped memory to the main memory.

The advantage of using this scheme is that the execution speed of the software portion of the application is independent of the memory used by the hardware. The disadvantages are: (a) the large communication costs of copying data from software to hardware and back, and (b) the creation of heavy bursts of traffic on the processor bus, which can potentially starve other devices that want to use it. Thus, to amortize the communication cost, the hardware-software partitioning has to be done in such a manner that communication between hardware and software is minimized.

\subsection{Shared Memory Based Schemes}

The other way hardware and software can interface is through shared memory (i.e. $M$ in Figure 6 is shared). This can be done by using shared memory with no local storage or shared memory with local storage. In the scheme with no local storage, variables and data structures in the shared memory are declared such that the compiler does not apply any memory optimizations and uses processor registers minimally (for example, by declaring them as volatile in the $\mathrm{C}$ code). Other forms of local storage like processor caches are also bypassed when accessing the shared memory. Data to be processed by software is always read from the shared memory (in hardware) and the results are immediately written back (no caching). Due to its simplicity, this scheme can be used with any processor.

In contrast, the shared memory with local storage scheme can only be used when the processor supports explicit instructions for flushing all local storage to memory. 


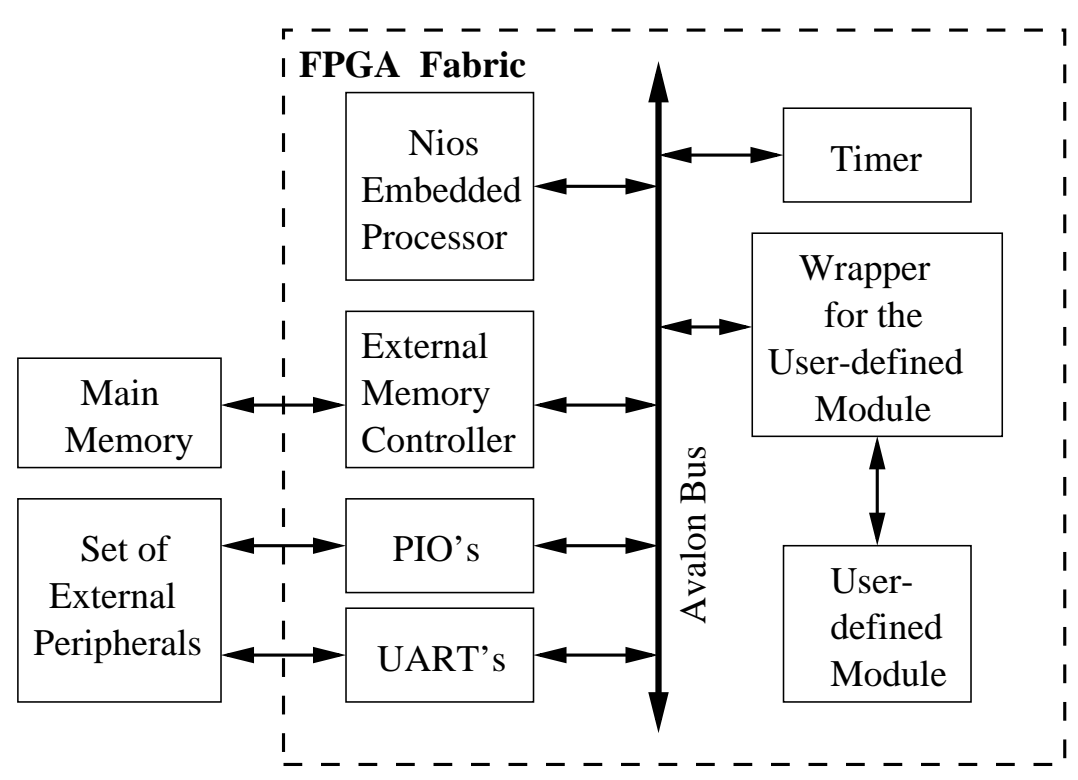

Figure 8. The Nios embedded system

The clear advantage of both these schemes is the zero data transfer (communication) cost between software and hardware. However, the shared memory with no local storage scheme has the additional advantage that it maintains data coherency since the data is not buffered inside the processor's local memory. But, this causes extra traffic on the processor bus whenever the software portion of the application is running. A disadvantage of both schemes is that a larger access time for mapped memory can degrade performance significantly. Thus, the performance with these schemes depends critically on the speed of the processor bus, the mapped memory and associated access logic.

\section{Experimental Setup and Results}

We used Altera's Nios development platform [19] for implementing the system. This platform consists of a soft core of the Nios embedded processor (no caches) to which various peripherals can be connected for customization. Once the system has been customized, it can be synthesized and mapped onto an FPGA based board provided by Altera. The user can use dedicated RAM block bits inside the FPGA to implement memories having two or fewer ports. The system level architecture is shown in Figure 8 where the user-defined module is the hardware depicted inside the FPGA in Figure 6. This entire system, consisting of the Nios processor and its standard peripherals, the main memory, the CPU bus and the user-defined module operate at a frequency of $33.33 \mathrm{MHz}$.

We synthesized application kernels using a parallelizing high level synthesis framework called SPARK [4]. This framework takes a behavioral description in $\mathrm{C}$ as input and generates synthesizable register-transfer level VHDL. SPARK applies a set of compiler, parallelizing compiler, and synthesis transformations to optimize the overall 
quality of the synthesized circuit, i.e., the circuit delay and area. The SPARK methodology is particularly useful for designs with complex control flow.

The generated RTL VHDL was synthesized using the logic synthesis tool, Leonardo Spectrum; the resultant netlist was mapped to the FPGA using the Altera Quartus tool. The portions of the application which were not mapped to hardware were compiled and mapped onto the processor on the FPGA platform using the compiler suite that is part of the Nios development toolkit.

\subsection{Case Study: MPEG-1 Prediction Block}

In this section, we present a case study using a multimedia application to evaluate the effectiveness of our mapping algorithm and to study the trade-offs between the three interfacing and communication schemes described in Section 5. We used the prediction block from the MPEG-1 multimedia application. The globals declared by this block were 53 integer variables and two integer arrays of 64 entries each; making a total of 181 integer data elements.

To begin with, three computationally intensive kernels in the application were identified using profiling information with the entire application running in software; each of these kernels consisted of nested loops with identical structures. High level synthesis of this loop structure was performed using our synthesis tool followed by the creation of a user-defined module consisting of the application logic for this loop structure, the memory controller, control logic and the bus interface logic. The resource allocation used for this design during high level synthesis was 1 ALU, 1 comparator and 1 shifter.

We used the Leonardo Spectrum logic synthesis tool to synthesize the user-defined module for the Altera FPGA. The results of this synthesis are shown in Table 1. This table gives the area of the synthesized circuit in terms of the logic cells, the maximum frequency that the synthesized hardware module can be executed at on the FPGA, and the amount of memory required for the hardware module in terms of RAM block bits. The first row shows synthesis results for the Nios system module without our user-defined module. The second row shows the results for the user-defined module described earlier in this section. This hardware module was obtained without applying our memory mapping technique. We could not integrate this design with the Nios embedded system because it was too big to fit inside the FPGA, given that the capacity of the FPGA was 8320 Logic Cells (LCs). Note that the maximum frequencies shown in Table 1 are only the estimates of the logic synthesis tool and were found to be slightly conservative.

Next, we applied the memory mapping algorithm to the 181 data elements in the design and came up with a new memory configuration. The constraint on the maximum number of ports per memory was set to two because the target FPGA could at the most support dual ported RAMs. Then, we made a new memory controller based on 


\begin{tabular}{|c|c|c|c|}
\hline HW Module & Area & Max Freq & RAM Bits \\
\hline \hline Nios System & $2800 \mathrm{LCs}$ & $47 \mathrm{MHz}$ & 26496 \\
\hline Unmapped User-defined & $9750 \mathrm{LCs}$ & $27 \mathrm{MHz}$ & 0 \\
\hline Mapped User-defined & $2450 \mathrm{LCs}$ & $32 \mathrm{MHz}$ & 2048 \\
\hline
\end{tabular}

Table 1. Logic synthesis results for different hardware modules mapped to an Altera APEX20KE EP20K200EFC484-2X FPGA target

the new memory map that was created after applying the memory mapping algorithm. This controller replaced the old one inside the user-defined module. We ran logic synthesis on the user-defined module again and obtained the results shown in the third row of Table 1.

When memory mapping was used, a 75\% reduction in area was observed because the logic cells used to implement the registers for storing the 181 data elements in the original case got freed up. These data elements got freshly mapped to regular banks of dual ported memory which was implemented using the FPGA's RAM block bits. A $19 \%$ increase in speed was also observed. This was primarily due to a significant reduction in the amount of multiplexing logic used after memory mapping was applied. The two tradeoffs involved were: (a) using 2048 extra RAM block bits and, (b) reduced flexibility in assigning addresses to mapped variables. The first tradeoff can practically be ignored because it constituted only $2 \%$ of the total RAM block bits available. The second tradeoff too was not very significant and called for only a slight manual effort to fix the application. Thus, the observed $75 \%$ reduction in area, along with the $19 \%$ increase in operating frequency, clearly shows the benefits of our memory mapping technique. As a result, we were also able to fit the hardware inside the FPGA easily.

For studying the three interfacing and communication schemes described in Section 5, the memory mapped version of the user-defined module was integrated with the Nios embedded system. To enable software to use the hardware kernels, a data structure was declared which contained all the global variables declared by the application. The structure also contained variables corresponding to each register inside the control logic block. These variables and registers were re-arranged as per the new memory map generated after performing memory mapping. The application accessed the hardware through a pointer to a data structure of this type (with the value of that pointer being set to the base address of the user-defined module). While implementing the shared memory with no local storage scheme, the data structure was simply qualified as volatile. Execution times were recorded using a timer peripheral that had been added to the system at the time of system integration.

Table 2 shows the breakup of execution times of various parts of the application based on a run of 500 iterations of the application. For the data transfer based scheme, the value given in parentheses for each kernel (under the HW column) indicates the cost of copying data from main memory to mapped memory and then vice-versa after 


\begin{tabular}{|c|c|c|c|c|}
\hline Scheme & Entity & $\mathrm{SW}(\mathrm{ms})$ & $\mathrm{HW}(\mathrm{ms})$ & Speedup \\
\hline \multirow{5}{*}{$\begin{array}{c}\text { Data } \\
\text { Transfer } \\
\text { Based }\end{array}$} & Kernel 1 & 110 & $12(17)$ & 9.2 \\
\hline & Kernel 2 & 110 & $12(17)$ & 9.2 \\
\hline & Kernel 3 & 108 & $12(17)$ & 9.0 \\
\hline & Misc & 42 & 96 & - \\
\hline & Total & 370 & 132 & 2.8 \\
\hline \multirow{5}{*}{$\begin{array}{l}\text { Shared } \\
\text { Memory } \\
\text { with no } \\
\text { Local } \\
\text { Storage }\end{array}$} & Kernel 1 & 178 & 12 & 14.8 \\
\hline & Kernel 2 & 178 & 12 & 14.8 \\
\hline & Kernel 3 & 183 & 12 & 14.8 \\
\hline & Misc & 42 & 60 & - \\
\hline & Total & 581 & 96 & 6.1 \\
\hline \multirow{5}{*}{$\begin{array}{c}\text { Shared } \\
\text { Memory } \\
\text { with } \\
\text { Local } \\
\text { Storage }\end{array}$} & Kernel 1 & 116 & 12 & 9.7 \\
\hline & Kernel 2 & 116 & 12 & 9.7 \\
\hline & Kernel 3 & 109 & 12 & 9.1 \\
\hline & Misc & 42 & 59 & - \\
\hline & Total & 383 & 95 & 4.0 \\
\hline
\end{tabular}

Table 2. Execution times for 500 iterations of various kernels of the application

execution. Miscellaneous cost represents the cost of executing everything other than the three kernels. Under the HW column, it represents the cost of running that portion of the application in software, which did not get mapped to hardware. It also includes all software overheads of using the hardware.

We see that the data transfer based scheme did not perform well with this application due to the overhead of copying significant amounts of data from the main memory to the mapped memory and back after execution. However, we might expect to see this scheme perform well when the computation is more time consuming compared to the communication time. The other two schemes performed much better because of the zero hardware software communication overhead associated with them and the fact that the bus speed was as high as the processor speed. Just as we would expect, as more and more of the application was run in software, the shared memory with local storage scheme performed progressively better than the shared memory with no local memory scheme because of the lack of full compiler optimizations in the latter.

The performances of Kernel 1 and Kernel 2 were always the same because the two were identical to each other while Kernel 3 was slightly different. In order to run Kernel 3 in hardware, the software needed to save a few variables in the main memory which it would restore after execution. Software also needed to perform a series of additions after Kernel 3 had finished executing in hardware. This cost of preparing to run Kernel 3 in hardware 


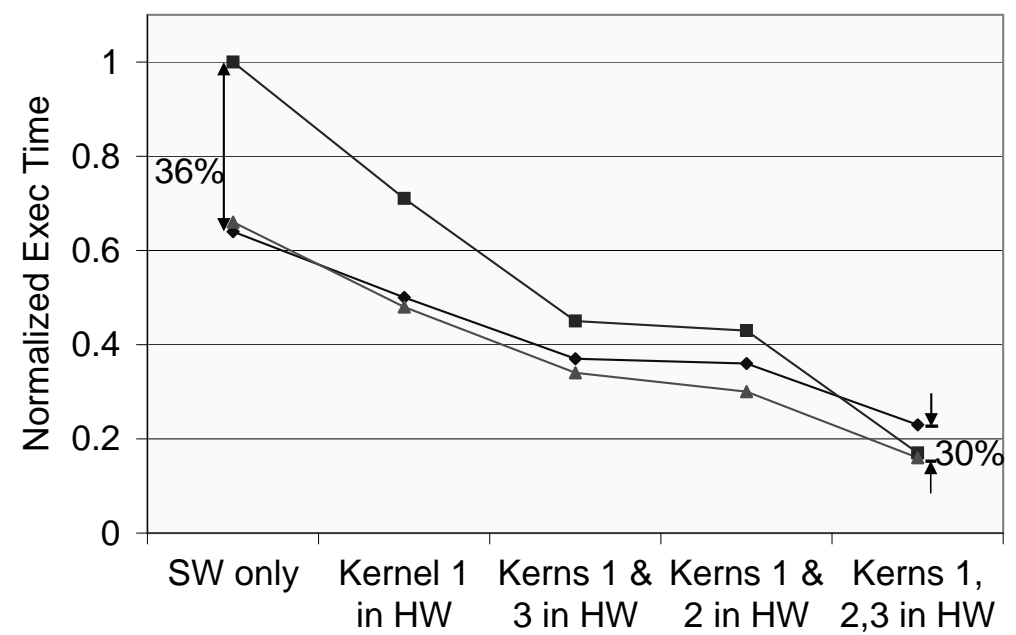

Data Transfer Based - Shared Memory with no Local Storage

Shared Memory with Local Storage

Figure 9. Execution times for 500 iterations of the application with different hardware software partitions

was accounted for in miscellaneous costs under the HW column. That explains why miscellaneous costs under hardware were observed to be more than the miscellaneous costs under software. Figure 9 shows the variation in execution time for each of the three schemes as the application shifts execution from software to hardware. This graph shows that the shared memory with local storage scheme performed the best from among the three, for various hardware software partitions. The data transfer based scheme was the fastest software only scheme; so taking it as the reference, the shared memory with local storage scheme gave a speedup of 3.9.

\section{Conclusions and Future Work}

We presented an interface synthesis approach that forms part of a hardware-software co-design methodology targeted at FPGA-based platforms. This approach is based on a novel memory mapping algorithm that maps the data elements operated on by both, software and hardware, to shared memories. These shared memories, thus, form a hardware interface between the hardware and software components of the application. We also presented three software schemes for bridging between the hardware interface and the application software. Results for experiments performed on an MPEG-1 application demonstrated an improvement in performance by a factor of 3.9 when three application kernels were accelerated in hardware using our interface synthesis approach. In future work, we want to validate our methodology for other FPGA platforms and develop a generalized hardware-software synthesis framework that includes task analysis and partitioning. We also want to investigate improvements to the heuristic used in the memory mapping algorithm. 


\section{Acknowledgments}

This project is funded by the Semiconductor Research Corporation under Task I.D. 781.001.

\section{References}

[1] A. Sangiovanni-Vincentelli and G. Martin. Platform-based design and software design methodology for embedded systems. IEEE Design and Test of Computers, December 2001.

[2] R.K. Gupta and G. De Micheli. Hardware-software cosynthesis for digital systems. IEEE Design and Test of Computers, September 1993.

[3] R. Camposano and R. Brayton. Partitioning before logic synthesis. In International Conference on Computer Aided Design, 1987.

[4] S. Gupta, N.D. Dutt, R.K. Gupta, and A. Nicolau. Spark : A high-level synthesis framework for applying parallelizing compiler transformations. In International Conference on VLSI Design, 2003.

[5] R. Camposano and W. H. Wolf. High-Level VLSI Synthesis. Kluwer Academic, 1991.

[6] P.Chou, R.Ortega, and G.Borriello. Interface co-synthesis techniques for embedded systems. In International Conference on Computer Aided Design, 1995.

[7] J. Daveau, G.F. Marchioro, T. Ben-Ismail, and A.A. Jerraya. Protocol selection and interface generation for hw-sw codesign. IEEE Transactions on VLSI Systems, March 1997.

[8] Z. Chen et al. Pilot - a platform-based hw/sw synthesis system for FPSoC. In Workshop on Software Support for Reconfigurable Systems, 2003.

[9] M.Balakrishnan, A.K.Majumdar, D.K.Banerji, J.G.Linders, and J.C.Majithia. Allocation of multiport memories in data path synthesis. IEEE Transactions on Computer Aided Design of Integrated Circuits and Systems, April 1988.

[10] L. Stok. Data path synthesis. Integration, The VLSI Journal, 1994.

[11] G. De Micheli. Synthesis and Optimization of Digital Circuits. McGraw Hill, 1994.

[12] I. Ouaiss and R. Vemuri. Hierarchical memory mapping during synthesis in FPGA-based reconfigurable computers. In Design Automation and Test in Europe, 2001. 
[13] F. Balasa, F. Catthoor, and H.De Man. Dataflow-driven memory allocation for multi-dimensional signal processing systems. In International Conference on Computer Aided Design, 1994.

[14] W.K.C. Ho and S.J.E. Wilton. Logical-to-physical memory mapping for FPGAs with dual-port embedded arrays. In International Workshop on FPL and Applications, 1999.

[15] D. Karchmer and J. Rose. Definition and solution of the memory packing problem for field-programmable systems. In International Conference on Computer Aided Design, 1994.

[16] D.D. Gajski, J. Zhu, R. Domer, A. Gerstlauer, and S. Zhao. SpecC: Specification Language and Methodology. Kluwer Academic, 2000.

[17] Synopsys Inc. SystemC Reference Manual. http://www.systemc.org.

[18] S. Vernalde, P. Schaumont, and I. Bolsens. An object oriented approach for hardware design. In IEEE Computer Society Workshop on VLSI, April 1999.

[19] The Altera Website. http://www.altera.com.

[20] The Xilinx Website. http://www.xilinx.com.

[21] E.G. Woutersen and S.H. Gerez. Some complexity results in memory mapping. In Third HCM BELSIGN Workshop, 1996. 